\title{
Misdiagnosis of tuberculosis in patients with lymphoma
}

\author{
B Puvaneswaran, B Shoba \\ Department of Internal Medicine, Ngwelezane Hospital, Empangeni, KwaZulu-Natal \\ B Puvaneswaran, MB BS, BSc (Hons) \\ B Shoba, MB BCh, FCP (SA)
}

Corresponding author: B Puvaneswaran (bhamini85@doctors.org.uk)

Background. Since 1970, the incidence of lymphoma, a potentially curable disease, has risen by $80 \%$ in the general population and in HIVpositive patients. Given its clinical similarities to tuberculosis (TB), lymphoma may be misdiagnosed and patients treated unnecessarily with potentially harmful TB medication.

Objectives. (i) To identify patients with a histological diagnosis of lymphoma who were previously misdiagnosed with TB; and (ii) to raise awareness of lymphoma as a differential diagnosis when TB has not been confirmed.

Method. A retrospective study was conducted at Ngwelezane Hospital in rural KwaZulu-Natal, which serves an estimated population of 3 million. Using clinic notes and a questionnaire for patients attending the lymphoma clinic, we identified patients who had undergone failed TB treatment in the 12 months before their histological confirmation of lymphoma.

Results. Twenty-one patients were included; 18 had been diagnosed with TB in the 12 months preceding the histological confirmation of lymphoma. All these patients subjectively reported TB treatment failure.

Conclusions. Delay in diagnosing lymphoma or its misdiagnosis is an important clinical problem in South Africa, with the condition often misdiagnosed as TB. This subjects patients to incorrect treatment and potential harm. We propose an algorithm for the work-up of patients presenting with lymphadenopathy $+/$ - constitutional symptoms, to assist diagnosis and management in resource-poor settings.

S Afr Med J 2013;103(1):32-33. DOI:10.7196/SAMJ.6093

Lymphomas - malignant disorders arising from cells of the lymphoid tissue - commonly present with lymphadenopathy and constitutional symptoms of fever, weight loss and night sweats. However, lymphomas can also affect extra-nodal sites, giving rise to atypical presentations. Owing to the varied clinical picture, especially in HIV-positive patients, symptoms may mimic other diseases, particularly tuberculosis (TB). ${ }^{1}$

In South Africa (SA), approximately 5.6 million people are living with HIV, with $\mathrm{TB}$ as the most serious opportunistic infection. ${ }^{2}$ The 2010 World Health Organization (WHO) report for SA revealed a treatment success rate of $73 \%$ for smear-positive TB and $64 \%$ for smear-negative/extra-pulmonary TB. ${ }^{2}$ These high treatment failure rates may be due to several factors, including incorrect diagnosis. TB also requires a prolonged treatment regimen with potential toxic side-effects, specifically liver toxicity. ${ }^{3}$ Therefore, despite difficulties in diagnosing TB, patients must be monitored regularly following treatment initiation, for clinical improvement and adverse events.

HIV has a strong association with non-Hodgkin's lymphoma (NHL), particularly aggressive B-cell lymphoma. ${ }^{4}$ HIV-positive patients are most susceptible when their viral loads are high and CD4 counts are low. Therefore, the use of highly active antiretroviral therapy (HAART) has dramatically reduced the incidence of HIV-associated NHL. ${ }^{5}$ In a study of a Swiss HIVpositive cohort, the incidence of HIV-associated NHL decreased from 13.6/1 000 per annum (p.a.) in 1993 - 1995 to 1.8/1 000 p.a. in 2002 - 2006; the beneficial effects remained for up to 10 years after HAART initiation, with overall survival nearing that of the general population. ${ }^{6}$

With an increasing incidence, lymphoma must be considered an important differential when TB cannot be confirmed. In developed ountries the diagnosis and management of TB can be monitored carefully, but this is not always feasible in resource-poor settings such as rural KwaZulu-Natal (KZN). We therefore aimed to identify patients with lymphoma who had been misdiagnosed with TB in this setting, to raise awareness of lymphoma and provide assistance to doctors working in rural areas.

\section{Method}

We retrospectively identified all patients who attended Ngwelezane Hospital's lymphoma clinic from 1 January to 30 April 2012. Histological diagnosis of lymphoma was confirmed from hospital records and patients were surveyed to establish whether they had been treated for TB in the 12 months prior to lymphoma diagnosis. Patients' subjective views regarding the success of TB treatment were noted. Data were anonymised and analysed using Microsoft Excel. Subjects gave informed written consent to participate, and ethical approval of the study was granted by the Ngwelezane Hospital ethics committee.

\section{Results}

Twenty-one patients with an histological diagnosis of lymphoma (Table 1) attended the clinic during the study period, including 15 females and 6 males. All patients presented with lymphadenopathy and/or constitutional symptoms of fever, weight loss or night sweats. Nineteen patients were found to have NHL, predominantly diffuse large B-cell lymphoma; only 2 had HL, 1 was HIV-positive. All subjects were tested for HIV within the preceding 12 months: 13 were HIV-positive, 11 of whom were receiving HAART.

Eighteen patients reported being diagnosed with TB in the year preceding histological confirmation of lymphoma; most reported that TB was diagnosed on medical history and examination alone. Three patients had given sputum samples for testing, but were unsure of the result. Only 7 patients reported being followed up at 1 month post treatment initiation. Patients had taken TB medication for a median duration of 5 months. All 18 patients reported little or no clinical improvement with TB treatment; none reported complications of TB treatment. Patients were referred to the lymphoma clinic after seeking 
Table 1. Cumulative representation of data from patients' clinic notes and questionnaire

\begin{tabular}{llllllll}
\hline Gender & LN/`B’ Symp & HIV status & HAART & NHL/HL & Diagnosed with TB & Started TB Rx & TB Rx success \\
\hline Female: 15 & Yes: 21 & HIV-positive: 13 & Yes: 11 & NHL: 19 & Yes: 18 & Yes: 18 & Yes: 0 \\
Male: 6 & No: 0 & HIV-negative: 8 & No: 2 & HL: 2 & No: 3 & No: 0 & No: 18
\end{tabular}

$\mathrm{LN}=$ lymphadenopathy; 'B' symptoms = fever, weight loss, night sweats; HAART = HIV-positive patients taking highly active antiretroviral therapy; NHL = non-Hodgkin’s lymphoma;
$\mathrm{HL}=$ Hodgkin's lymphoma; TB = tuberculosis; Rx = treatment.

further medical help or following incidental recognition of associated symptoms on admission to the medical ward at Ngwelezane Hospital.

\section{Discussion}

Lymphadenopathy and constitutional symptoms are found in several disease states. While we found all patients in our cohort to be symptomatic with one or both features, none had a confirmed $\mathrm{TB}$ culture before starting treatment and few were adequately followed up. Because of the growing burden of TB in SA as a result of the HIV pandemic, WHO and SA guidelines suggest empirical treatment of TB, particularly in HIV-positive patients. ${ }^{78}$ However, these guidelines stress the importance of sample culture testing, with follow-up investigations and reviews of treatment response. Unfortunately, this is difficult in resource-poor and disease-heavy settings, due to factors including:

- large numbers of patients

- poor management of rural health clinics

- failure of patients to take responsibility for their health.

In our cohort, all patients diagnosed with TB subjectively reported no symptomatic improvement despite a median treatment duration of 5 months. This must be considered when explaining high levels of TB treatment failure reported by WHO in 2011: $27 \%$ and 36\% in smearpositive and smear-negative/extra-pulmonary cases, respectively. ${ }^{2}$

Unfortunately, as most patients were diagnosed with TB at rural clinics, we were unable to obtain formal clinical notes to access results of clinical findings and investigations. Treatment success was therefore based solely on patients' subjective accounts. Furthermore, due to the recent introduction of the lymphoma clinic at Ngwelezane Hospital, few patients had histological confirmation, resulting in a small sample size. Further work is needed to confirm our findings of lymphoma misdiagnosis, or at the very least, of diagnosis delay.

The delayed diagnosis or misdiagnosis of lymphoma is an important clinical problem in SA. If TB treatment is initiated empirically, it must be monitored for clinical response and side-effects. Our study results reveal that this is unfortunately not common practice in rural SA. We therefore propose an algorithm (Fig. 1) to assist healthcare providers to diagnose lymphoma and monitor TB treatment.

Most hospitals in the study region have access to fine needle aspiration for acid-fast bacilli and lymph node biopsy. It may be impractical to perform lymph node biopsies in all culture-negative patients; however, it is known that TB responds rapidly in HIVpositive patients. ${ }^{9}$ We therefore suggest that clinicians initiate treatment empirically with close patient monitoring. Patients failing to respond to treatment after 1 month must undergo lymph node biopsy.

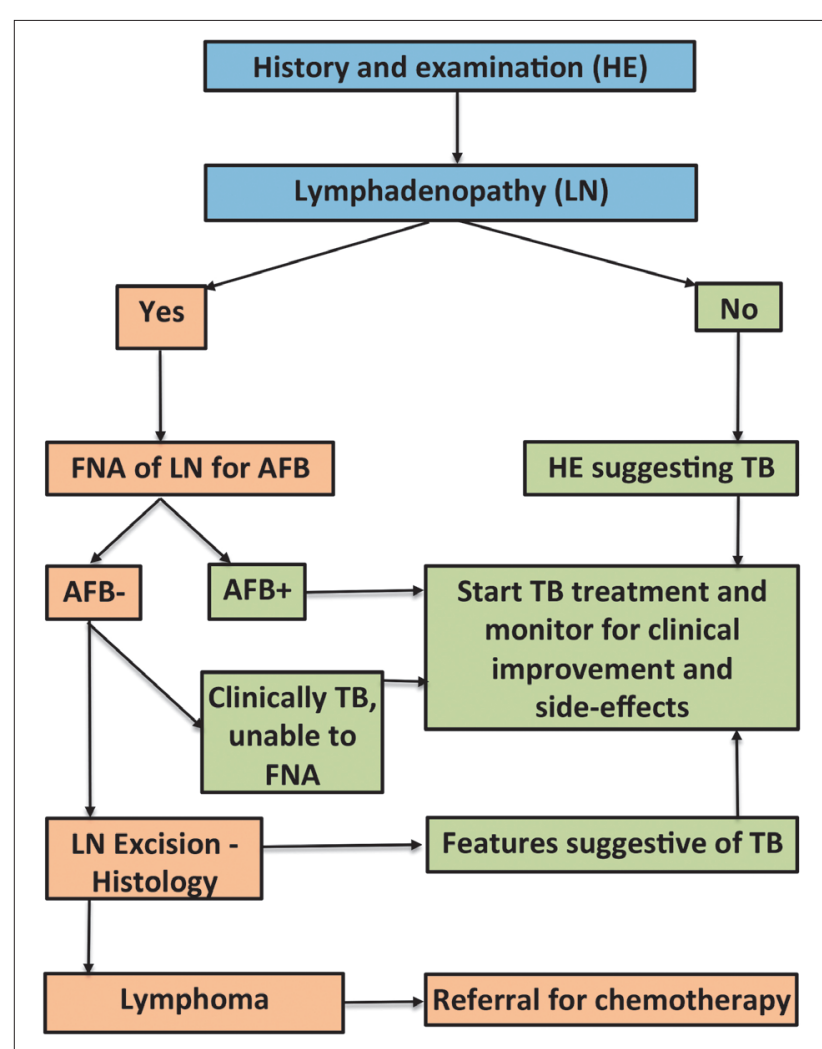

Fig. 1. Algorithm for diagnosing lymphoma.

References

1. Al-Mobeireek AF, Arafah M, Siddiqui N. An African male with cough, haemoptysis, weight loss and hypercalcaemia: TB or not TB? Eur Respir J 2002;20(4):1060-1063. 1. [http://dx.doi.org/10.1183/0903 1936.02.00632002]

2. Baddeley A, Dias HM, Falzon D, et al. WHO Report 2011: Global Tuberculosis Control. Geneva: World Health Organization, 2011.

Pukenyte E, Lescure FX, Rey D, et al. Incidence of and risk factors for severe liver toxicity in HIV-infected patients on

4. Chiu BC, Weisenburger DD. An update of the epidemiology of non-Hodgkin's lymphoma. Clin Lymphoma 2003;4(3):161-168. [http://dx.doi.org/10.3816/CLM.2003.n.025]

5aplan L. HIV-associated lymphoma. Best Pract Res Clin Haematol 2012;25(1):101-117. [http://dx.doi org/10.1016/j.beha.2012.01.001]

6. Polesel J, Clifford GM, Rickenbach M, et al. Non-Hodgkin lymphoma incidence in the Swiss HIV Cohort Study before and after highly active antiretroviral therapy. AIDS 2008;22(2):301-306. [http://dx.doi. org/10.1097/QAD.0b013e3282f2705d]

7. The South African National Tuberculosis Control Programme. Practical Guidelines. Pretoria Department of Health, 2004. http://www.kznhealth.gov.za/chrp/documents/Guidelines/Guidelines\%20 Department of Health, 2004. http://www.kznhealth.gov.za/chrp/documents/Guidelines/Guidelines\%20

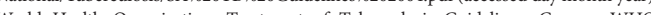

8. World Health Organization. Treatment of Tuberculosis Guidelines. Geneva: WHO, 2010. http:// whqlibdoc.who.int/publications/20109789241547833_eng.pdf

. Wilson D, Nachega J, Morroni C, Chaisson R, Maartens G. Diagnosing smear-negative tuberculosis using case definitions and treatment response in HIV-infected adults. Int J Tuber Lung Dis 2006;10(1):31-38.

Accepted 19 September 2012. 